\title{
Fingerprint Image Enhancement using Filtering Techniques
}

$\mathrm{E}$ xtracting minutiae from fingerprint images is one of the most important steps in automatic fingerprint identification and classification. Minutiae are local discontinuities in the fingerprint pattern, mainly terminations and bifurcations. Most of the minutiae detection methods are based on image binarization while some others extract the minutiae directly from gray-scale images. In this work we compare these two approaches and propose two different methods for fingerprint ridge image enhancement. The first one is carried out using local histogram equalization, Wiener filtering, and image binarization. The second method uses a unique anisotropic filter for direct gray-scale enhancement. The results achieved are compared with those obtained through some other methods. Both methods show some improvement in the minutiae detection process in terms of time required and efficiency.

(C) 2002 Elsevier Science Ltd. All rights reserved.

\section{Shlomo Greenberg, Mayer Aladjem and Daniel Kogan \\ Electrical and Computer Engineering Department Ben-Gurion University of the Negev, Beer-Sheva, Israel E-mail:shlomog@ee.bgu.ac.il}

\section{Introduction}

Fingerprints are today the biometric features most widely used for personal identification. Fingerprint recognition is one of the basic tasks of the Integrated Automated Fingerprint Identification Service (IAFIS) of the most famous police agencies [1]. A fingerprint pattern is characterized by a set of ridgelines that often flow in parallel, but intersect and terminate at some points. The uniqueness of a fingerprint is determined by the local ridge characteristics and their relationships [2,3]. Most automatic systems for fingerprint comparison are based on minutiae matching [4]. Minutiae characteristics are local discontinuities in the fingerprint pattern and represent the two most prominent local ridge characteristics: terminations and bifurcations. A ridge termination is defined as the point where a ridge ends abruptly, while ridge bifurcation is defined as the point where a ridge forks or diverges into branch ridges (Figure 1). A typical fingerprint image contains about 40-100 minutiae [2].

An automatic fingerprint image matching process, which enables a personal identification, strongly depends on comparison of the minutiae points of interest (MPOI) and their relationships. Reliable automatic extraction of these MPOI is a critical step in fingerprint classification.

The performance of minutiae extraction algorithms relies heavily on the quality of the fingerprint images [2]. The ridge structures in poor-quality fingerprint images are not always well defined and, hence, cannot be correctly detected. This might result in the creation of spurious minutiae and the ignoring of genuine minutiae. Therefore, large errors in minutiae localization may be introduced [2]. Examples of poorquality fingerprint images are shown in Figure 2. In 


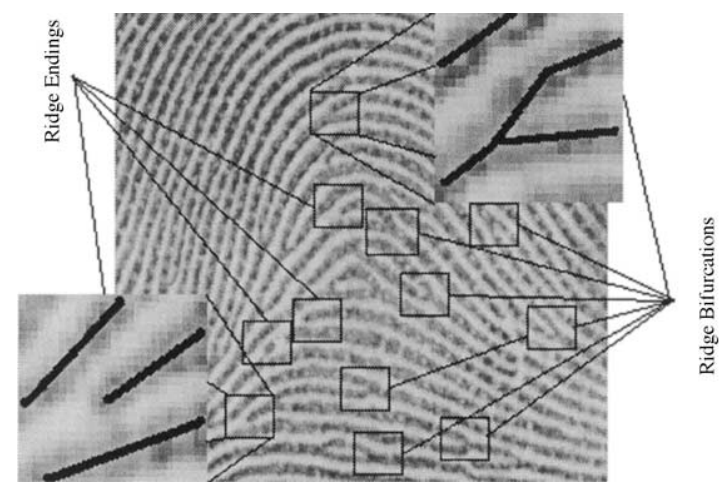

Figure 1. Examples of minutiae (ridge ending and bifurcation) in a fingerprint image.

order to ensure robust performance of a minutiae extraction algorithm, an enhancement algorithm, which can improve the clarity of the ridge structures, is necessary $[2,5]$.

Most of the fingerprint image enhancement methods proposed in the literature are applied to binary images, while some others operate directly on gray-scale images $[1,6,7]$. Concerning these two approaches, this work proposes two methods for fingerprint image enhancement. The first one is carried out using local histogram equalization, Wiener filtering, and image binarization.

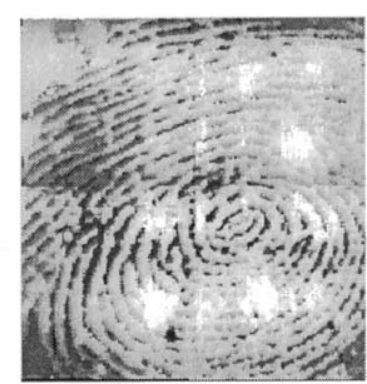

(a)

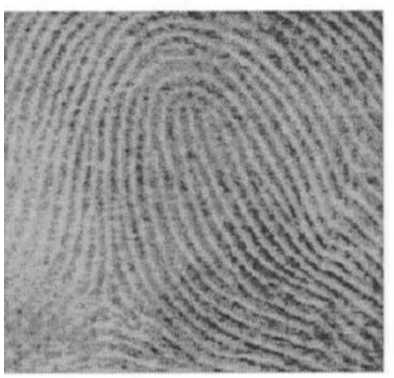

(c)

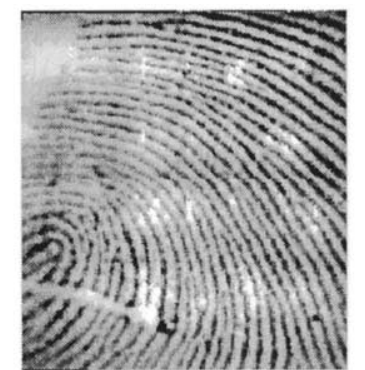

(b)

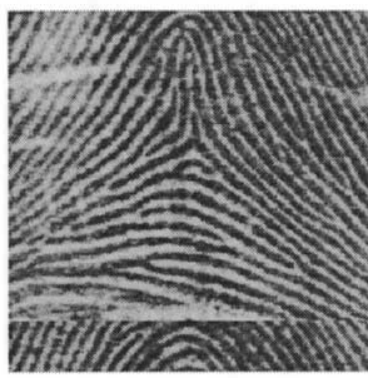

(d)
Figure 2. Examples of poor-quality fingerprint images due to: noisy acquisition device (a), (b) and variation in impression conditions (c), (d), results in corrupted ridgelines.
The second method uses a unique anisotropic filter for direct gray-scale enhancement.

The paper is organized as follows. The following section addresses the main steps for our binarization approach (see section, A binarization-based method). Then we present some modifications to the Gabor-based technique introduced by Hong [2], and propose a fast direct gray-scale fingerprint enhancement algorithm (see section, Direct gray-scale enhancement approaches) based on a unique anisotropic filter. The section Experimental Results presents the results of a comparative study of our approaches and the methods described by Maio [1] and Hong [2]. Finally, in the last section some conclusions are drawn. Some preliminary results of our work were presented by Greenberg [8]. This paper contains a more thorough analysis and more complete results.

\section{Fingerprint enhancement}

Fingerprint enhancement can be conducted on either binary ridge images or gray-scale images. The binarization process may cause loss of information about the true ridge structure and it has inherent limitations [2]. Different techniques for gray-level fingerprint images enhancement have been proposed [6,7] assuming that the local ridge frequency and orientation can be reliably estimated. However, this assumption is not valid for poor-quality fingerprint images. Although, other decomposition methods [2,5], which apply a bank of Gabor filters to the input fingerprint images, can obtain reliable orientation estimation even for corrupted images, they are computationally expensive.

Steerable filters are a class of filters, in which a filter of arbitrary orientation is synthesized as a linear combination of a set of "basis filters' [9]. Steerable-scalable kernels roughly shaped like Gabor functions have the advantage that they can be specified and computed easily [10]. However, current steerability approaches suffer from the consequences of the uncertainty principle, and in order to achieve a high orientational resolution a huge number of basis filters must be used [11].

Hong [2] proposed a fast enhancement algorithm, which can adaptively improve the clarity of ridge and valley structures of input fingerprint images based on the estimated local ridge orientation and frequency.

We present some improvement to the Hong [2] method by using a unique anisotropic filter, adapted 
to fingerprint images. Instead of using both local ridge orientation and local frequency information, only the orientation information is used in our approach.

Our proposed anisotropic filter, which eliminates the need to estimate local frequency information, can replace the Gabor filter.

Almansa used diffusion techniques, which are based on a multi-scale analysis called the scale-space representation, and applied an iterative process for local features estimation $[12,13]$. Another kind of structureadaptive anisotropic filtering technique has been proposed by Yang [14]. Instead of using local gradients as a means of controlling the anisotropism of filters, it uses both a local intensity orientation and an anisotropic measure to control the shape of the filter.

Although the filters proposed by Yang and Almansa are both structure-adaptive anisotropic filters, they still significantly differ. We employed Yang's anisotropic filter, which does not use diffusion techniques, for fingerprint enhancement.

The proposed enhancement algorithm is faster and efficient as well.

\section{A binarization-based method}

In some binarization-based approaches the binarization and thinning process are preceded by a smoothing operation. Moayer [15] used a smoothing operation based on convolution with a Gaussian $5 \times 5$ pixels mask, in order to regularize the starting image. We propose an enhancement process, which combines filters and noise reduction techniques for pre- and post-processing as well. The proposed scheme is based on adaptive histogram equalization for contrast expansion, followed by Wiener filtering for noise reduction [16]. The binarization process is applied using an adaptive threshold based on the local intensity mean. Then a thinning process is carried out through the algorithm presented by Baruch [17], which provides good results on fingerprints. Finally, morphological filtering is applied to eliminate artifacts in noisy regions and to fill some gaps in valid ridgelines. The main stages of our proposed enhancement process conducted on binary ridge fingerprint images are shown in Figure 3.

Contrast enhancement. Histogram equalization defines a mapping of gray levels $p$ into gray levels $q$ such that the distribution of gray levels $q$ is uniform [16]. This

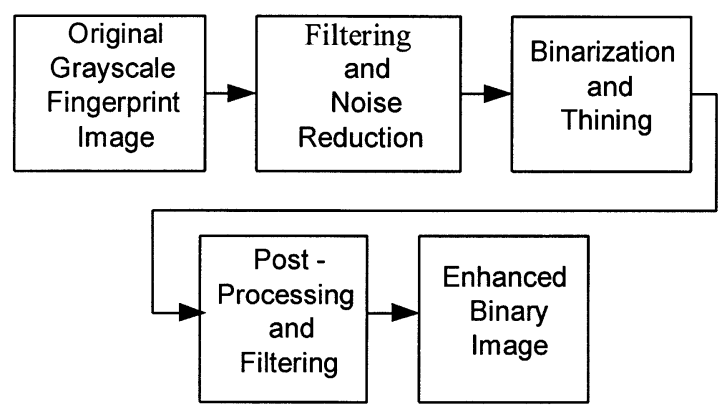

Figure 3. Filtering and binarization-based enhancement process.

mapping stretches contrast (expands the range of gray level) for gray levels near histogram maxima. Since contrast is expanded for most of the image pixels, the transformation improves the detectability of many image features. The probability density function of a pixel intensity level $r_{k}$ is given by

$$
p_{r}\left(r_{k}\right)=\frac{n_{k}}{n}
$$

where $0 \leq r_{k} \leq 1, k=0,1, \ldots, 255, n_{k}$ is the number of pixels at intensity level $r_{k}$ and $n$ is the total number of pixels. The histogram is derived by plotting $p_{r}\left(r_{k}\right)$ against $r_{k}$. A new intensity $s_{k}$ of level $k$ is defined as

$$
s_{k}=\sum_{j=0}^{k} \frac{n_{j}}{n}=\sum_{j=0}^{k} p_{r}\left(r_{j}\right)
$$

We apply the histogram equalization locally by using local windows of $11 \times 11$ pixels. This results in expanding the contrast locally, and changing the intensity of each pixel according to its local neighborhood. Figure 4(b) presents the improvement in image contrast obtained by applying the local histogram equalization.

Wiener filtering noise reduction. We propose using a pixelwise adaptive Wiener method for noise reduction. The filter is based on local statistics estimated from a local neighborhood $\eta$ of size $3 \times 3$ of each pixel, and is given by the following equation:

$$
w\left(n_{1}, n_{2}\right)=\mu+\frac{\sigma^{2}-v^{2}}{\sigma^{2}}\left(I\left(n_{1}, n_{2}\right)-\mu\right)
$$

where $v^{2}$ is the noise variance, $\mu$ and $\sigma^{2}$ are the local mean and variance and $I$ represents the gray-level intensity in $n_{1}, n_{2} \in \eta$. Figure 5(a) shows the result of the Weiner filtering.

Binarization and thinning. The operation that converts a gray-scale image into a binary image (Figure 5(b)) is 


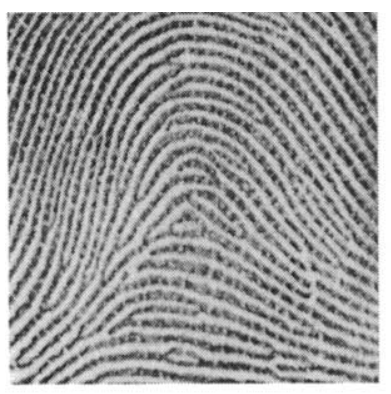

(a)

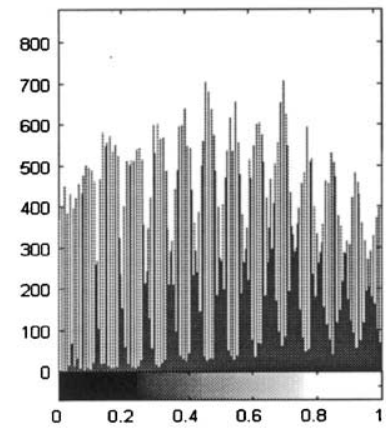

(c)

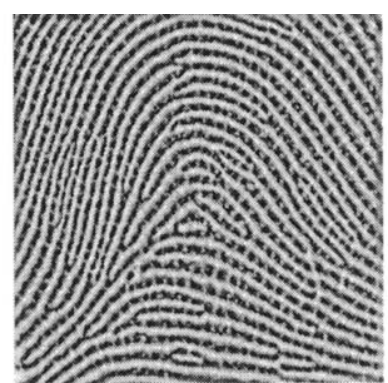

(b)

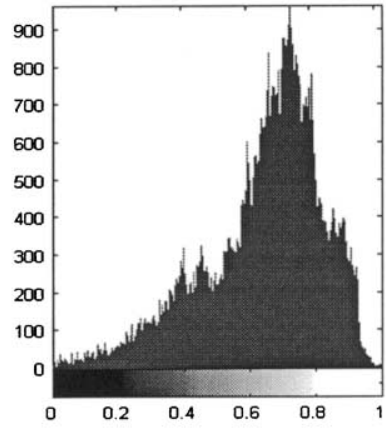

(d)
Figure 4. Histogram equalization: original image (a) and its histogram (c), and after equalization (b) (d), respectively.

known as binarization. We carried out the binarization process using an adaptive thresholding. Each pixel is assigned a new value ( 1 or 0 ) according to the intensity mean in a local neighborhood $(13 \times 13$ pixels $)$, as follows:

$$
I_{\text {new }}\left(n_{1}, n_{2}\right)= \begin{cases}1 & \text { if } I_{\text {old }}\left(n_{1}, n_{2}\right) \geq \text { Local Mean } \\ 0 & \text { otherwise }\end{cases}
$$

Thinned (one pixel thickness) ridgelines are obtained using morphological thinning operations as indicated in Figure 7(a).

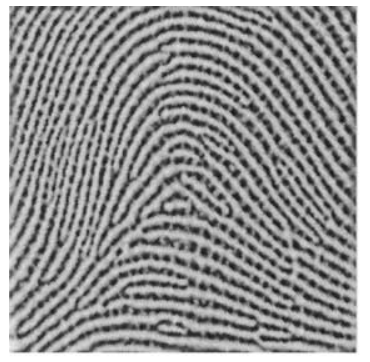

(a)

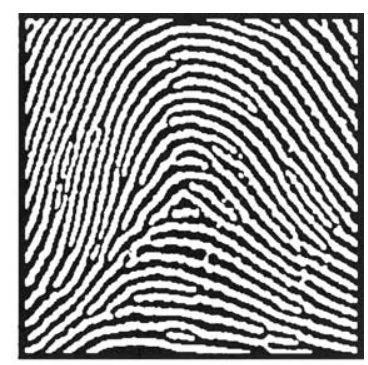

(b)
Figure 5. Wiener filtering result using local neighborhood of $3 \times 3$ pixels (a) and a binary image (b).

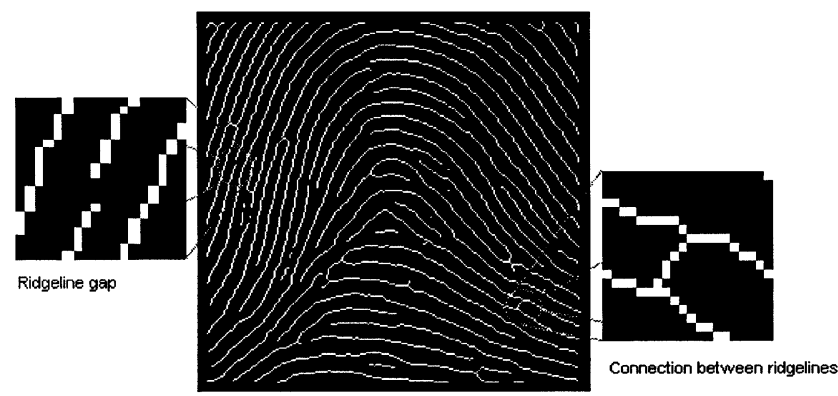

Figure 6. Typical noise in the thinned binary image: false connections between ridgelines (right), and ridgeline gaps (left).

Post-processing and binary filtering. Figure 6 shows two typical kinds of noise, which can appear in a thinned binary image: false ridgeline connections and gaps within a true ridgeline. False ridgeline connections are almost perpendicular to the local ridge direction, and empirically found to be of length less than 10 pixels. Our algorithm automatically removes lines with similar characteristics (Figure 7). Filling the space between each of the two end-points that belongs to the same ridge direction eliminates gaps of up to 15 pixels, within a true continuous ridgeline. The maximum allowed gap is determined empirically due to a trade-off between false ridgelines and missed detection of true long gaps.

\section{Direct gray-scale enhancement approaches}

Maio and Maltoni [1] propose a technique, based on ridgeline following, where the minutiae are extracted directly from gray-scale images. Hong [2] introduced a fast fingerprint enhancement algorithm based on a Gabor filter. In this section we propose a direct grayscale enhancement method, which has been simulated by Hong [2]. First, we suggest some modifications of

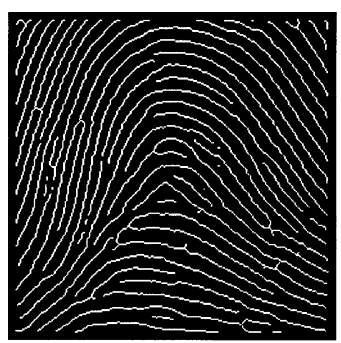

(a)

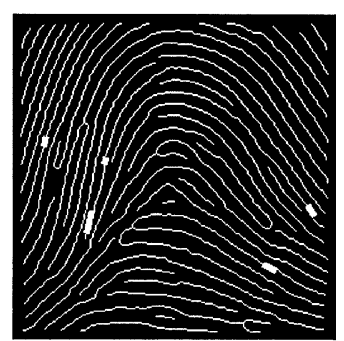

(b)
Figure 7. Post-processing: thinned binary image (a) and removing false ridges and filling gaps (b). 
Hong's original algorithm, and then we propose a fast direct gray-scale fingerprint enhancement based on an anisotropic filter.

A modification of the Gabor-based algorithm. In this section we suggest some improvements to Hong's [2] Gaborbased filtering technique. The main steps of Hong's algorithm include normalization, local orientation estimation, local frequency estimation and filtering. A bank of Gabor filters, which is tuned to local ridge orientation and ridge frequency, is applied to the ridge and valley pixels in the normalized input fingerprint image to obtain an enhanced fingerprint image. The filters are used as band-pass filters to remove the noise and preserve true ridge/valley structures. We implemented this algorithm for comparison purposes, introducing some modification.

First, an alternative scheme, based on local gradient operations [1], is used for more precise orientation estimation. Fine tuning of some parameters in the original algorithm result in an efficient and more robust algorithm. The selection of $\delta_{x}^{2}$ and $\delta_{y}^{2}$ involves a trade-off between robustness and spurious ridges. The values of $\delta_{x}^{2}$ and $\delta_{y}^{2}$ were equally set of 4.0 based on empirical data [2]. This selection gives the same importance both to ridge direction and to the direction perpendicular to it. In order to decrease the standard deviation of the Gaussian envelope in the direction perpendicular to the ridge direction $\left(\delta_{y}^{2}\right)$ we set those values to 4.0 and 3.0, respectively. The new parameter setting creates less spurious ridges and makes the filter more robust to noise. For a given resolution, the value of the ridge frequency in a local neighborhood lies in a certain range [2]. By cutting down the valid frequency range, we avoid wrong estimation of the frequency in blocks which do not form a well-defined frequency. Finally, for better definition of the block center, in the ridge frequency algorithm we divide the normalized image into an odd block of size $(15 \times 15)$ instead of $(16 \times 16)$.

Enhancement using a unique anisotropic filter. In this section we present a new direct gray-scale approach based on a unique anisotropic filter. A structureadaptive anisotropic filtering technique is proposed by Yang [14] for image filtering. Instead of using local gradients as a means of controlling the anisotropism of filters, it uses both a local intensity orientation and an anisotropic measure to control the shape of the filter. We modified this anisotropic filter by shaping the filter kernel and applied it to fingerprint images. The basic idea is that the filter kernel is shaped or scaled according to local features within a given neighborhood. The filter kernel applied at each point $x_{0}$ is defined as follows [14]:

$$
\begin{aligned}
& k\left(x_{0}, x\right)=\rho\left(x-x_{0}\right) \\
& \quad \exp \left\{-\left[\frac{\left(\left(x-x_{0}\right) \cdot n\right)^{2}}{\sigma_{1}^{2}\left(x_{0}\right)}+\frac{\left(\left(x-x_{0}\right) \cdot n_{\perp}\right)^{2}}{\sigma_{2}^{2}\left(x_{0}\right)}\right]\right\}
\end{aligned}
$$

where $n$ and $n_{\perp}$ are mutually normal unit vectors, and $n$ is parallel to the ridge direction. The shape of the kernel is controlled through $\sigma_{1}^{2}\left(x_{0}\right)$ and $\sigma_{2}^{2}\left(x_{0}\right), \rho$ satisfies the condition $\rho(x)=1$ when $|x|<r$, and $r$ is the maximum support radius. We modified the original filter to a band-pass filter type in order to adapt it to fingerprint images. The proposed anisotropic filter has the following general form:

$$
\begin{aligned}
& h\left(x_{0}, x\right)=V+S \cdot \rho\left(x-x_{0}\right) \\
& \quad \exp \left\{-\left[\frac{\left(\left(x-x_{0}\right) \cdot n\right)^{2}}{\sigma_{1}^{2}\left(x_{0}\right)}+\frac{\left(\left(x-x_{0}\right) \cdot n_{\perp}\right)^{2}}{\sigma_{2}^{2}\left(x_{0}\right)}\right]\right\}
\end{aligned}
$$

where $V$ and $S$ are parameters, which must be adjusted to a specific application. Applying a 2D Fourier transform to Eqn (6), we obtain the filter's frequency response:

$$
\begin{aligned}
H(u, v)= & V \cdot 4 \pi^{2} \delta(u, v)+\frac{1}{\pi \beta} S\left(\frac{\sin (u r)}{u} * \exp \left\{-\frac{u^{2}}{4 \beta}\right\}\right) \\
& \cdot\left(\frac{\sin (v r)}{v} * \exp \left\{-\frac{v^{2}}{4 \beta}\right\}\right) \\
\beta= & \left(\frac{\cos \theta}{\sigma_{1}\left(x_{0}\right)}\right)^{2}+\left(\frac{\sin \theta}{\sigma_{2}\left(x_{0}\right)}\right)^{2}
\end{aligned}
$$

where $\theta$ is the local pattern orientation, $r$ is the kernel's maximal support radius and * stands for convolution. We adjusted the filter empirically by setting the filter parameters to $V=-2$ and $S=10$. The filter frequency response has band-pass filter characteristics, and was found to be effective in removing noise, while preserving the local ridge frequency of the fingerprint image. The frequency bands transferred by the filter include almost all typical local ridge frequencies that lie within a certain range for a given image resolution [2]. Figure 8 shows a comparison of impulse and frequency responses for the original and the modified anisotropic filters. Both filters have directional Gaussian-shaped kernels in the space domain. However, in the frequency domain the original filter shows low pass (one peak in the center) filter characteristics, while the modified anisotropic filter represents band-pass filter characteristics (two peaks 


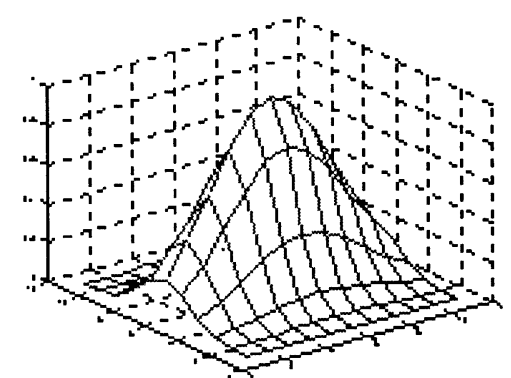

(a)

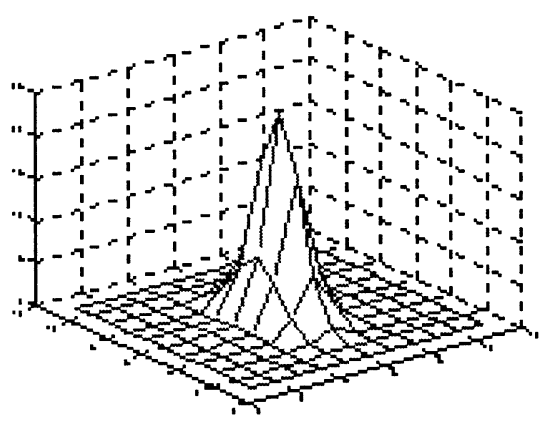

(c)

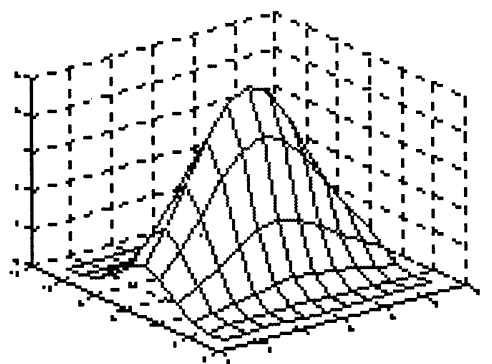

(b)

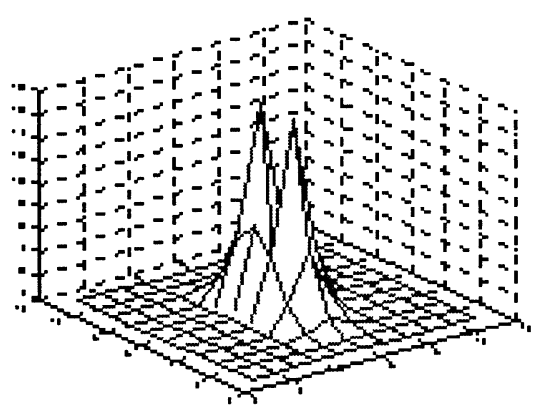

(d)

Figure 8. Comparison of impulse and frequency response between the original (a) and the modified (b) anisotropic filters $(V=-2, S=10)$. Both filters have an $11 \times 11$ pixels kernel size. Both filters have a directional Gaussian-shaped kernel in a space domain (a) (b). However, they are different in the frequency domain: (c) the original anisotropic filter shows low-pass (one peak in the center) filter characteristics, while (d) the modified anisotropic filter represents band-pass filter characteristics (two peaks).

symmetrically located around the center). An accurate estimation of the space constants $\sigma_{1}^{2}\left(x_{0}\right)$ and $\sigma_{2}^{2}\left(x_{0}\right)$ in Eqn (6) is actually local frequency estimation. However, no accurate estimation of these constants is needed. We set them empirically to be as follows: $\sigma_{1}^{2}\left(x_{0}\right)=4$ and $\sigma_{2}^{2}\left(x_{0}\right)=2$. This setting produced a Gaussian-shaped kernel filter, with a 1:2 ratio between the two kernel axes.

When a ridgeline edge is encountered, the kernel is deformed into a ellipse with the major axis aligned parallel with the edge (Figure 9). Therefore, smoothing is performed along but not across the ridgeline. Our approach is quite similar to the Gabor-based technique, as both are using oriented filters. By applying our filter, only orientation information is required. In our enhancement algorithm we replaced the Gabor filter used by Hong [2] with the proposed anisotropic filter, which eliminates the need to estimate the local frequency information. This makes our algorithm faster than the one proposed by Hong. The main advantages of the anisotropic filter are its nondependence on ridge frequency and its robustness against noise and distortions in the ridge frequency image.

\section{Experimental results}

In order to quantitatively assess the performance of the fingerprint enhancement algorithm the following error criteria are defined:

Dropped minutiae: Minutiae that were not found in the neighborhood of true minutiae.

False minutiae: Minutiae that were found in the region not containing true minutiae.

Exchanged minutiae: Minutiae differing from the true minutiae type in the same image region.

\section{Comparison of binarization-based methods}

Here we compare our binarization-based method with some other similar techniques. Maio [1] reports the average error percentage obtained with four different schemes (B-E) based on binarization and thinning. We refer to Maio ([1, Figure 20]), and use fingerprints from the same sample set in order to compare those four schemes to our binarization scheme, named $\mathrm{F}$ (see 


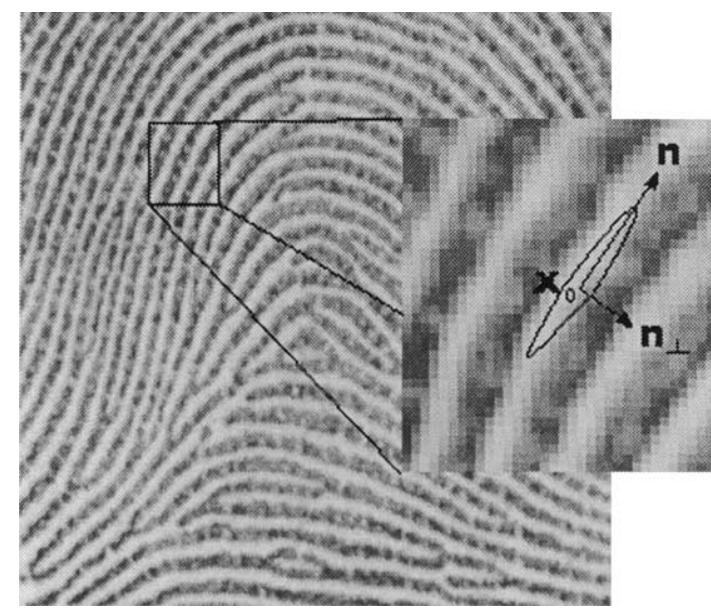

Figure 9. Controlling the shape of an anisotropic filter kernel.

section, A binarization-based method). The samples set consists of 10 fingerprints taken from NIST, an FBI sample and through an opto-electronic device. Figure 10 shows the average error percentage obtained with the five different approaches. The results are reported in terms of undetected (dropped), non-existent (false), and type exchanged (exchanged) minutiae [1].

\section{Comparison of direct gray-scale methods}

In this section we compare the enhancement results conducted on gray-scale images by four different methods: the direct gray-scale miniature detection $(\mathrm{G})$ used by Maio [1], the modified Gabor-based filtering $(\mathrm{H})$ (see section, A modification of the Gabor-based algo-

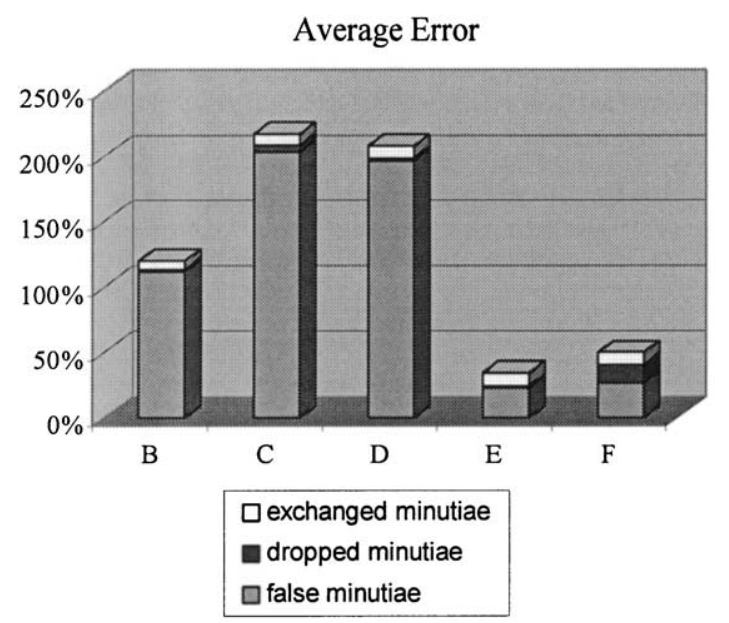

Figure 10. Comparison of 5 binarization-based schemes. rithm), the proposed anisotropic filter (I) and the original anisotropic filter (J) (see section, Enhancement using a unique anisotropic filter).

Figure 11 compares the average error percentage obtained by applying the four different filters $(\mathrm{G}, \mathrm{H}, \mathrm{I}, \mathrm{J})$ for the same sample set as in the section Comparison of binarization-based methods. The average error percentage is expressed in terms of false, dropped and exchanged minutiae.

Figure 12 shows enhancement results obtained for poor-quality fingerprint images, which contain regions that do not form a well-defined local ridge frequency. These regions are mostly encountered in the neighbourhood of fingerprint image singular points: core and delta [1]. The enhancement was carried out with the Gaborbased algorithm (see section, A modification of the Gabor-based algorithm), and with the anisotropic filter (see section, Enhancement using a unique anisotropic filter). Both the original and the improved anisotropic filters outperform the Gabor-based filters for those regions which contain singular points.

Figure 13 compares the enhancement results obtained by applying the two different filter techniques, the anisotropic filter and the Gabor filter, to some fingerprint images from the sample set.

Figure 14 shows the minutiae extracted from some input images and from the corresponding filtered

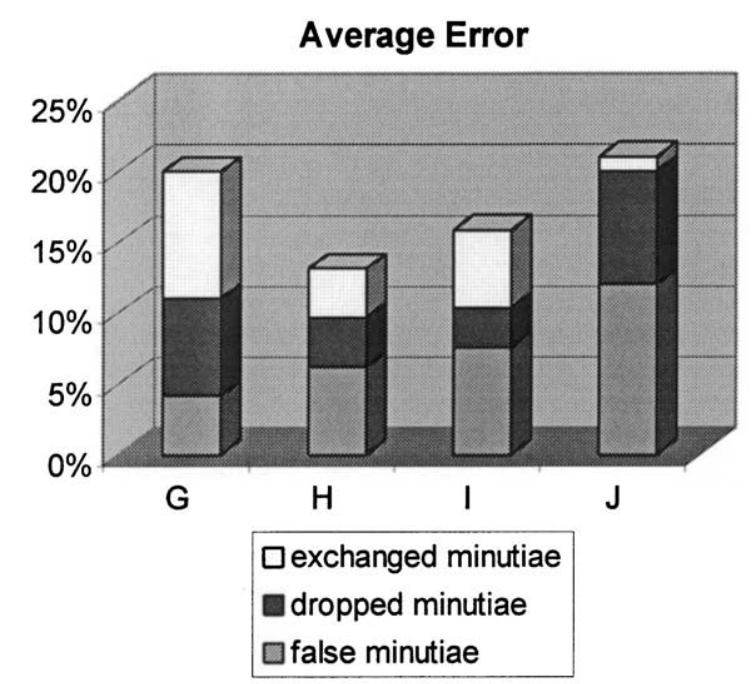

Figure 11. Performance comparison of the gray-scale approaches: $\mathrm{G}$ - Gabor filter, $\mathrm{H}$ - modified Gabor filter, I-modified anisotropic filter and $\mathbf{J}$ - the original anisotropic filter. 


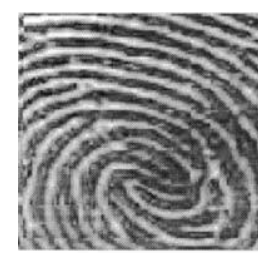

(a)

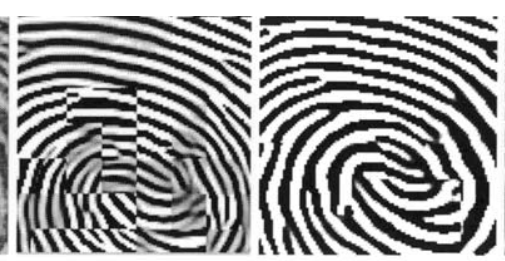

(b)

(c)

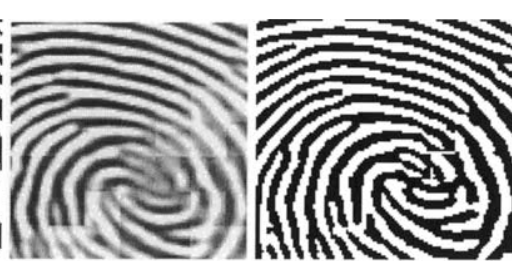

(d)

(e)

Figure 12. Enhancement results for poor fingerprint images: original image (a); enhanced image using Gabor filter (b), modified Gabor filter (c), original anisotropic filter (d) and the modified anisotropic filter (e).

images. It can be seen that the use of the proposed enhancement algorithm results in a better minutiae set that in turn will lead to improvements in matching performance.
Table 1 shows the wall time for different stages of the Gabor-based enhancement algorithm [2] and the total time on a Pentium $200 \mathrm{MHz}$ PC. The enhancement algorithm based on the anisotropic filter does not

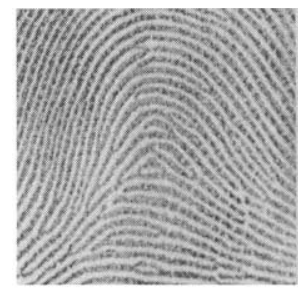

(a)

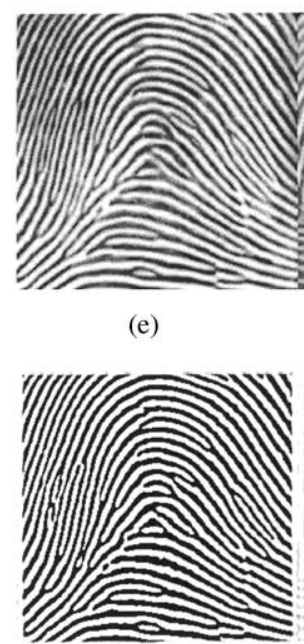

(i)

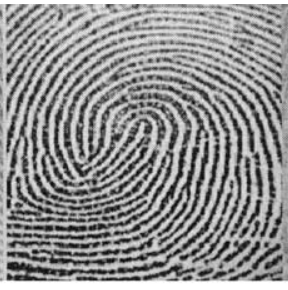

(b)

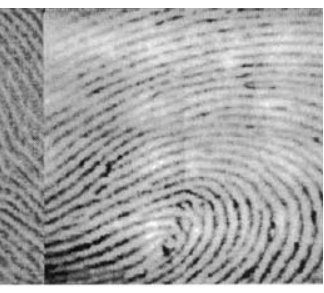

(c) (d)

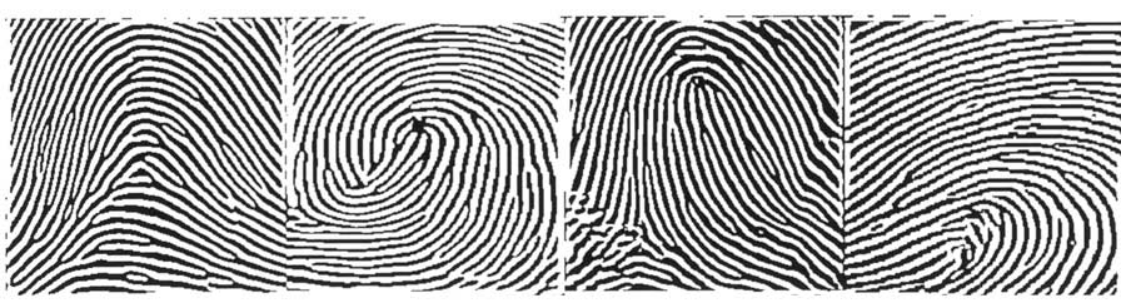

(m)

(n)

(o)

(p)

Figure 13. Enhancement results of applying different filters to fingerprint images: (a)-(d) original fingerprint images and after enhancement by using (e)-(h) original anisotropic filter, (i)-(l) the modified anisotropic filter and (m)-(p) the improved Gabor filter. 


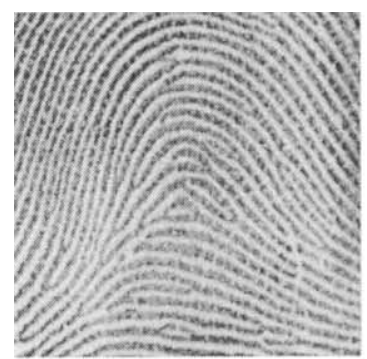

(a)

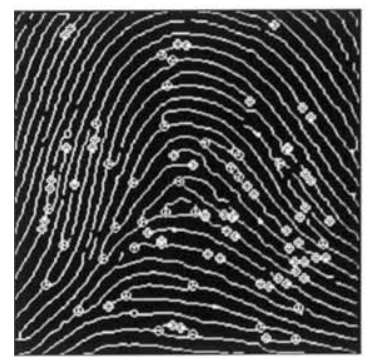

(c)

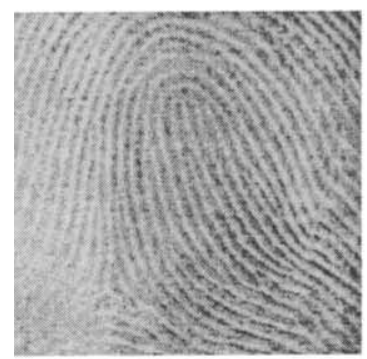

(e)

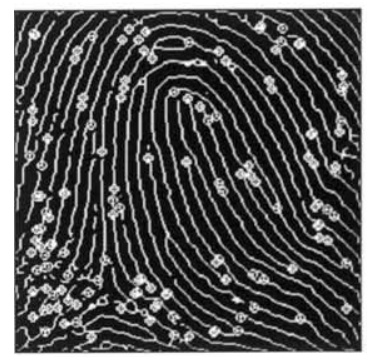

(g)

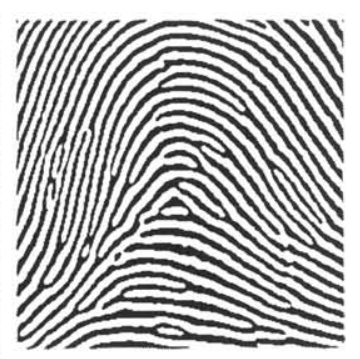

(b)

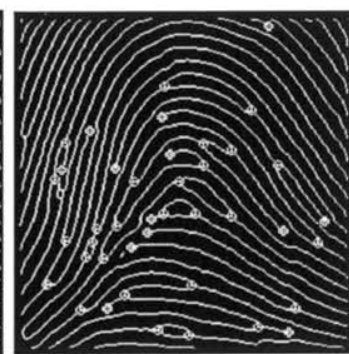

(d)

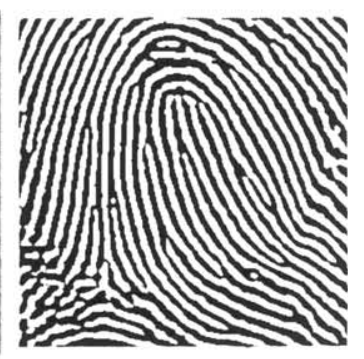

(f)

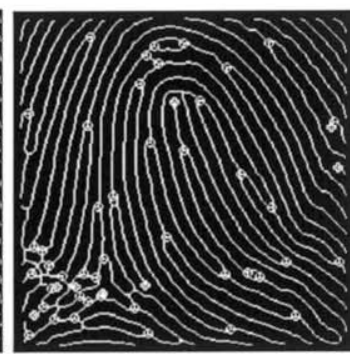

(h)
Figure 14. Minutiae extracted from some input images and from the corresponding filtered images. Input images (a) (e), their filtered images (b) (f) and images with extracted minutiae (c) (d) (g) (h), correspondingly.

require the estimation of the local ridge frequency information. Therefore, it saves about $4 \%$ of the processing effort compared to the Gabor-based enhancement algorithm.
Table 1. The wall time of the Gabor-based enhancement algorithm on an Pentium 200 MHZ PC (taken from [2, Table 2])

\begin{tabular}{lccccc}
\hline $\begin{array}{l}\text { Normali- } \\
\begin{array}{l}\text { zation } \\
(\mathrm{s})\end{array}\end{array}$ & $\begin{array}{c}\text { Orientation } \\
(\mathrm{s})\end{array}$ & $\begin{array}{c}\text { Frequency } \\
(\mathrm{s})\end{array}$ & $\begin{array}{c}\text { Region } \\
\text { mask } \\
(\mathrm{s})\end{array}$ & $\begin{array}{c}\text { Filtering } \\
(\mathrm{s})\end{array}$ & $\begin{array}{c}\text { Total } \\
(\mathrm{s})\end{array}$ \\
\hline 0.11 & 0.14 & 0.09 & 0.07 & 2.08 & 2.49 \\
\hline
\end{tabular}

\section{Summary and conclusions}

The techniques based on direct gray-scale enhancement perform better than approaches which require binarization and thinning as intermediate steps. The average error percentage, in terms of dropped, exchanged and false minutiae, as produced by our binarization approach $\mathrm{F}$, is considerably lower than the errors produced by approaches B-D and comparable to the errors produced by approach E. The modified Gabor filter $\mathrm{H}$ performs better than the original scheme introduced by Hong [2], especially for poor-quality images with corrupted ridges and blocks with singular points.

Although the anisotropic filter has been already applied to fingerprints [12], to the best of our knowledge this is the first time that a structure-adaptive anisotropic filter, which does not use the diffusion technique, has been applied to fingerprint images. Improvements were made to the original anisotropic filter in order to adjust it to fingerprint images. The resulting filter shows robustness against noise, while restoring the true ridge/ valley of the fingerprint image. The performance comparison shows that an anisotropic filter outperforms the Gabor filter for poor-quality fingerprint images. Moreover, applying the proposed anisotropic filter to fingerprint images eliminates the need for accurate estimation of local frequency information, as implemented by other Gabor-based filters. The proposed enhancement scheme is faster and efficient as well.

\section{References}

1. Maio, D. \& Maltoni, D. (1997) Direct Gray-Scale Minutiae Detection in Fingerprints. IEEE Transactions on Pattern Analysis and Machine Intelligence 19: 27-40.

2. Hong, L., Wan, Y. \& Jain, A. (1998) Fingerprint Image Enhancement: Algorithm and Performance Evaluation. IEEE Transactions on Pattern Analysis and Machine Intelligence 20: 777-789.

3. Lee, H.C. \& Gaensslen, R.E. (1991) Advanced in Fingerprint Technology. New York: Elsevier. 
4. Hollingum, J. (1992) Automated Fingerprint Analysis Offers Fast Verification. Sensor Review 12: 12-15.

5. Hong, L., Jain, A.K., Pankanti, S. \& Bolle, R. (1996) Fingerprint Enhancement. Proceedings of the First IEEE WACV, Sarasota, FL, pp. 202-207.

6. O'Gorman, L. \& Nickerson, J.V. (1989) An Approach to Fingerprint Filter Design. Pattern Recognition 22: 29-38.

7. Sherlock, D., Monro, D.M. \& Millard, K. (1994) Fingerprint Enhancement by Directional Fourier Filtering. IEE Proceedings on Visual Imaging Signal Processing, 141: $87-94$.

8. Greenberg, S., Aladjem, M., Kogan, D. \& Dimitrov, I. (2000) Fingerprint Image Enhancement using Filtering Techniques. 15th International Conference on pattern Recognition, Barcelona, vol. III, pp. 326-329.

9. Freeman, W.T. \& Adelson, E.H. (1991) The Design and Use of Steerable Filters. IEEE Transactions on Pattern Analysis and Machine Intelligence 13: 891-906.

10. Perona, P. (1992) Steerable-Scalable Kernels for Edge Detection and Junction Analysis. Image and Vision Computing 10: 663-672.
11. Yu, W., Daniilidis, K. \& Sommer, G. (2001) Approximate Orientation Steerability Based on Angular Gaussians. IEEE Transactions on Image Processing 10: 193-205.

12. Almansa, A. \& Lindeberg, T. (2000). Fingerprint Enhancement by Shape Adaptation of Scale-Space Operators with Automatic Scale Selection. IEEE Transactions on Image Processing 9: 2027-2042.

13. Weickert, J. (2001) Applications of nonlinear diffusion in image processing and computer vision. Acta Mathematica Universitatis Comenianae 70: 33-50.

14. Yang, G.Z., Burger, P., Firmin, D.N. \& Underwood, S.R. (1996) Structure Adaptive Anisotropic Filtering. Image and Vision Computing 14: 135-145.

15. Moayer, B. \& Fu, K. (1986) A Tree System Approach for Fingerprint Pattern Recognition. IEEE Transactions on Pattern Analysis and Machine Intelligence 8: 376-388.

16. Gonzales, R.C. \& Woods, R.E. (1993) Digital Image Processing. New York, USA: Addison-Wesley.

17. 17. Baruch, O. (1998) Line Thinning by Line Following. Pattern Recognition Letters 8: 271-276. 